\title{
Effect of intermolecular disorder on the intrachain charge transport in ladder-type poly $(p$-phenylenes $)$
}

\author{
P. Prins, ${ }^{1}$ F. C. Grozema, ${ }^{1}$ J. M. Schins,${ }^{1}$ T. J. Savenije, ${ }^{1}$ S. Patil, ${ }^{2}$ U. Scherf,${ }^{2}$ and L. D. A. Siebbeles ${ }^{1}$ \\ ${ }^{1}$ Opto-Electronic Materials Section, DelftChemTech, Delft University of Technology, Mekelweg 15, $2629 \mathrm{JB}$ Delft, The Netherlands \\ ${ }^{2}$ Department of Chemistry and Institute for Polymer Technology, Bergische Universität Wuppertal, Wuppertal, Germany
}

(Received 12 August 2005; revised manuscript received 22 November 2005; published 11 January 2006)

\begin{abstract}
We have studied the real and imaginary parts of the complex intrachain mobility of charge carriers on solid samples of ladder-type polymers using time-resolved microwave conductivity measurements. Experiments on samples with a different average polymer chain length show that the motion of charge carriers is limited by the chain ends. The experimental results can be described by one-dimensional diffusive motion along the polymer backbone. The intrachain mobility deduced for an infinitely long ladder-type polymer chain (with no barriers to charge transport such as defects or conjugation breaks) subject to interchain interactions is $30 \mathrm{~cm}^{2} / \mathrm{V} \mathrm{s}$. This value is 20 times lower than the intrachain mobility found for charges along infinitely long isolated ladder-type polymer chains in dilute solution. Thus we find that interchain interactions in solid samples severely decrease the intrachain charge carrier mobility. However, the intrachain mobility of $30 \mathrm{~cm}^{2} / \mathrm{V} \mathrm{s}$ is more than four orders of magnitude higher than mobility values obtained from time of flight measurements reported in the literature. Hence, the performance of ladder-type polymers in optoelectronic devices can be significantly improved.
\end{abstract}

DOI: 10.1103/PhysRevB.73.045204

PACS number(s): 72.80.Le, 72.20.Jv

\section{INTRODUCTION}

The optoelectronic properties of conjugated polymers are of interest due to their applications in devices, such as organic light-emitting diodes, photovoltaic cells, and fieldeffect transistors. ${ }^{1-4}$ Using solution-processable polymers as semiconducting compounds opens the way to flexible electronics and enables cheap and easy production of devices. ${ }^{3,5}$ Moreover, the ongoing miniaturization of electronic components rapidly approaches the fundamental limit of photolithography. Therefore, it is of interest to study the ultimate size limit for organic electronics. Single molecules are the smallest components that are likely to be functional in integrated circuits. ${ }^{6-9}$ Conjugated polymers can be used as interconnecting molecular wires between molecular scale electronic components.

For the applications mentioned above, the mobility of charge carriers is a key factor determining the performance of the electronic devices. The mobility of charges in a onedimensional, ordered (crystalline) material is determined by their effective mass $m_{e f f}=2 \hbar^{2} / W d^{2}$, where $W$ is the bandwidth of the conduction band and $d$ is the length of the repeating unit or unit cell of the material. Using the typical length of one monomer $(3.5 \AA)$ for the size of the unit cell and a bandwidth as typically calculated for perfectly ordered polymer chains ${ }^{10}(3-5 \mathrm{eV})$, an effective mass of about onefourth of the free electron mass is obtained for conjugated polymers. Since this effective mass is comparable to the effective mass for charges in inorganic semiconductors, the mobility of charge carriers along a perfectly ordered polymer chain is expected to be in the same order of magnitude as the mobility found for inorganic semiconductors, i.e., in the order of $1000 \mathrm{~cm}^{2} / \mathrm{V} \mathrm{s} .{ }^{11}$ Indeed a value of $600 \mathrm{~cm}^{2} / \mathrm{V} \mathrm{s}$ has been found for the mobility of holes along isolated ladder-type poly(paraphenylene) chains in dilute solution by time-resolved microwave conductivity (TRMC) measure- ments. ${ }^{12}$ However, the values found for the device mobility in conjugated polymers, range from $10^{-7}-10^{-2} \mathrm{~cm}^{2} / \mathrm{V} \mathrm{s}$, for derivatives of poly(para-phenylenvinylene), poly(thienylenevinylene), poly(thiophene), and ladder-type poly(paraphenylene). ${ }^{4,13-15}$ These values are several orders of magnitude lower than expected on the basis of the arguments above.

This difference in charge carrier mobility is likely to be due to a combination of device-specific properties and intrinsic material properties. Contact effects are the most important of the device-specific properties and determine the performance of organic optoelectronic devices to a large extent. ${ }^{12,16}$ Two examples of intrinsic properties of organic materials that limit the charge carrier mobility are interchain transport and charge transport over grain boundaries (boundary junctions between different relatively ordered domains within the sample). Both processes are expected to be significantly slower than intrachain transport and depend strongly on the material morphology on a supramolecular scale. Furthermore, the intrachain charge carrier mobility can be limited by disorder along the polymer chains. Effects that cause such intrachain disorder include torsion of the polymer backbone and the presence of chemical defects that can act as barriers to charge transport. ${ }^{17-19}$ In addition, intrachain disorder can originate from interchain interactions. Places where the backbones of neighboring polymer chains closely interact might act as localization sites that are energetically favorable for charge carriers. Therefore, the potential energy landscape encountered by a charge moving along the backbone of a polymer chain can be significantly altered by the presence of neighboring polymer chains.

In this contribution we describe a time-resolved microwave conductivity study of charge transport in solid samples of ladder-type polymers. The ladder-type polymer (see Fig. 1) is a structurally well defined polymer with no torsional disorder, since it is restricted to a planar geometry by a 


\section{Phe-LPPP}

$<n>=13,16,35$

$\mathrm{R}_{1}=n$-hexyl

$\mathrm{R}_{2}=t$-butyl

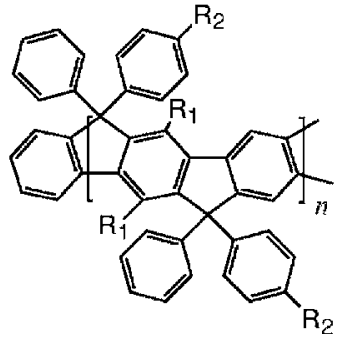

Me-IJPPP

$<n>=54$

$\mathrm{R}_{1}=n$-hexyl

$\mathrm{R}_{2}=n-\mathrm{dec} y \mathrm{l}$

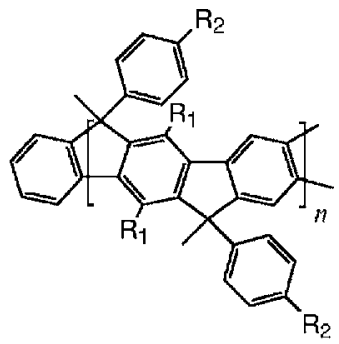

FIG. 1. Chemical structure of the ladder-type polymers studied and the average number of repeat units per polymer chain $(\langle n\rangle)$ calculated from the number averaged molecular weight. bridging carbon atom. Such a structure is optimal for charge transport because the electronic coupling between neighboring units is maximal for a planar structure. ${ }^{18}$ The structural conformation of the ladder-type polymer in a device setup is expected to be similar to the conformation of the polymer in dilute solutions, where the intrachain mobility was found to be $600 \mathrm{~cm}^{2} / \mathrm{V} \mathrm{s}^{20}$ Comparison of the intrachain mobility of the ladder-type polymer in solid samples where interchain effects can play a role to the value found for isolated polymer chains, gives information about the effect of interchain interactions on the intrachain charge transport properties. With the TRMC technique, the motion of charge carriers is probed by microwaves, in this way the difficulties that arise in a device setup are circumvented. ${ }^{21}$ In contrast to dc measurements, the microwave conductivity is dominated by the most efficient charge transport process. The intrachain motion of charges in solid polymer samples is expected to be much faster than the motion of charges from chain to chain. Therefore the microwave conductivity will mainly be determined by the intrachain charge transport properties. TRMC experiments on solid polymer samples provide insight into the charge transport along polymer chains in an environment that resembles the surroundings of the polymer chains in a device setup.

The one-dimensional mobility of charge carriers in disordered materials depends on the radial frequency of the (probing) electric field $(\omega)$, as described by $\mathrm{Kubo}^{22}$

$$
\mu(\omega)=-\frac{e \omega^{2}}{2 k_{B} T} \int_{0}^{\infty} \Delta^{2}(t) e^{-i \omega t} d t,
$$

where $\Delta^{2}(t)$ is the mean squared displacement of the charge carrier as a function of time. In this expression $e$ denotes the elementary charge, $k_{B}$ is Boltzmann's constant, and $T$ is the temperature. The frequency dependence of the charge carrier mobility can be understood qualitatively by taking into account the length scales that are probed at different frequencies. The mobility determined at a high probing frequency will be sensitive to the motion of charge carriers on a relatively short length scale, whereas the mobility observed at low (or zero) frequency will be sensitive to the displacement of the charge carriers over a longer distance. In a disordered material the motion of charges can be hindered by barriers to charge transport. While the charge carriers are relatively mobile in between the barriers, their motion can be severely hindered as they move over larger distances. Therefore, the hindrance of charge carrier motion in disordered materials is more pronounced at low probing frequencies.
At a nonzero frequency the charge carrier mobility is a complex quantity as can be seen from Eq. (1). We study this complex mobility $\mu(\omega)=\mu_{r e}(\omega)+i \mu_{i m}(\omega)$ of charge carriers diffusing along polymer chains in the presence of a weak oscillating electric field $E(\omega)=E_{o} \cos (\omega t)$, which can be represented by $E(\omega)=\operatorname{Re}\left(E_{o} e^{i \omega t}\right)$. Here $E_{o}$ denotes the amplitude and $\omega$ the (radial) frequency of the probing electric field. The velocity of charges expressed in terms of these (complex) quantities is given by

$$
\begin{aligned}
v(\omega) & =\operatorname{Re}\left[\mu_{r e}(\omega) E_{o} e^{i \omega t}+\mu_{i m}(\omega) E_{o} e^{i(\omega t+\pi / 2)}\right] \\
& =\mu_{r e}(\omega) E_{o} \cos (\omega t)-\mu_{i m}(\omega) E_{o} \sin (\omega t) .
\end{aligned}
$$

The term containing the real part of the mobility corresponds to the velocity of charge carriers in phase with the oscillating electric field, while the term that contains the imaginary part of the mobility represents the motion out of phase with the electric field. The field-induced drift velocity of a charge performing normal Gaussian diffusion along an ordered, infinitely long polymer chain with no barriers to charge transport is completely in phase with the probing electric field. In this case the imaginary part of the complex mobility equals zero and the motion of charges is completely described by the real part of the mobility. Disorder along the polymer chain leads to a hindrance of charge carrier motion and thus to motion out of phase with the probing field. Therefore, a nonzero imaginary part of the complex conductivity will be observable when the charge carrier motion is hindered by the presence of barriers to charge transport.

From the TRMC measurements we obtain direct information about the real and imaginary parts of the complex mobility for ladder-type polymers. Systematic variation of the polymer chain length provides information about the extent to which the motion of charge carriers is hindered by the chain ends. The intrachain mobility of charge carriers moving along an infinitely long polymer chain is obtained from the TRMC measurements using an analysis that assumes one-dimensional diffusive motion along the polymer backbone and infinitely high barriers to charge transport at the chain ends. A comparison of the present results on solid samples with results on isolated polymer chains in dilute solutions shows that the intrachain mobility is negatively affected by the disordered environment that results from interchain interactions.

\section{EXPERIMENT}

\section{A. Ladder type polymer}

The polymers studied are the ribbonlike ladder-type poly ( $p$-phenylenes) shown in Fig. $1 .^{23-25}$ The polymer chains 

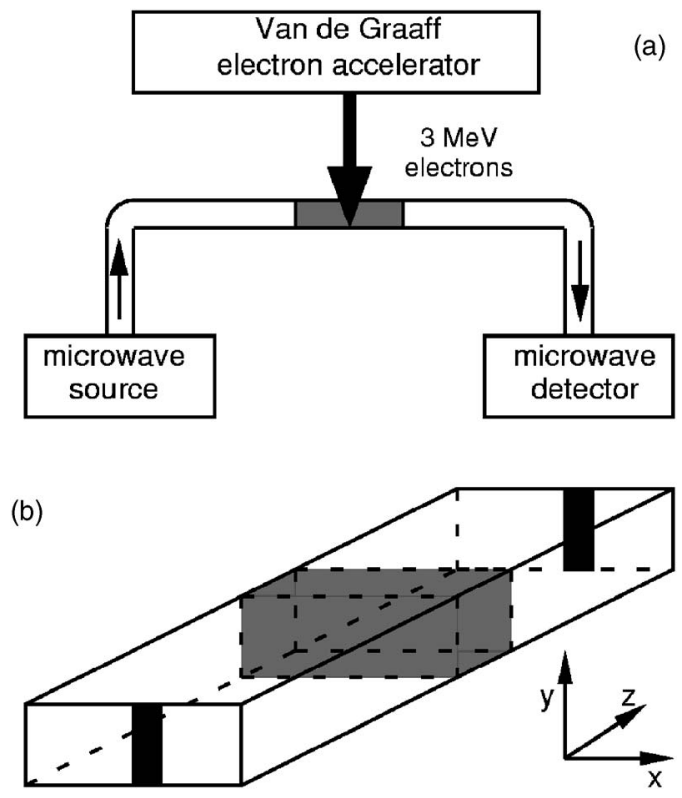

FIG. 2. (a) Schematic representation of the experimental setup. The microwaves are generated by the source and directed onto the resonant cavity depicted in gray by a microwave wave guide. The transmitted power $\left(P_{t}\right)$ is detected as a function of frequency and time after irradiation of the polymer sample with $3 \mathrm{MeV}$ electrons from the Van de Graaff electron accelerator. (b) Schematic representation of the resonant cavity that is confined by two metal strips that serve as irises. The ladder-type polymer (depicted in gray) is placed in a polyethylene container $(x \times y \times z=5 \mathrm{~mm} \times 2.5 \mathrm{~mm}$ $\times 24 \mathrm{~mm})$.

are restricted to a planar geometry by a bridging carbon atom. Hence, intrachain disorder due to torsion of the polymer backbone is not present for these polymers. The chain length is distributed according to a Flory distribution, from which chains shorter than five repeat units have been removed by solvent extraction. Samples with an average chain length of $13,16,35$, and 54 repeat units as calculated from the number averaged molecular weight, are studied.

\section{B. Generation of charge carriers}

The experiments described were performed on solid samples of about $3 \mathrm{mg}$ of the ladder-type polymer in poly(ethylene) containers that were placed in a microwave resonant cavity (see Fig. 2). Charge carriers were generated by irradiation of the polymer with a pulse of $3 \mathrm{MeV}$ electrons from a Van de Graaff electron accelerator. The duration of the electron pulse was varied between 0.5 and 50 ns. Irradiation with high-energy electrons causes tracks of ionizations in the polymer sample, leading to a close to uniform distribution of positive and negative charges in the polymer material. The number density of charge carriers created initially by irradiation of the sample with the $3 \mathrm{MeV}$ electron pulse is given by

$$
n_{\text {in }}=E_{d e p} / E_{p},
$$

where $E_{d e p}$ is the energy deposited in the sample per unit volume during the pulse and $E_{p}$ is the pair formation energy, i.e., the average energy deposition involved in the creation of one electron-hole pair. The energy deposited in the sample per unit volume can be obtained from the measured irradiation dose $\left(D_{i r r}\right.$ in Gray, $\left.1 \mathrm{~Gy}=1 \mathrm{~J} / \mathrm{kg}\right)$ which was determined using thin film radiochromic dosimetry. The pair formation energy of the polymer backbone can be approximated by the empirical relation derived by $\mathrm{Alig}^{26}$ that relates the pair formation energy (in eV) in (inorganic) semiconductors to their band gap $\left(E_{g}\right.$ in $\left.\mathrm{eV}\right)$

$$
E_{p, b b} \approx 2.73 E_{g}+0.5
$$

This relation is found to provide reasonable estimates for the pair formation energy for materials varying from inorganic semiconductors with a band gap as low as $1 \mathrm{eV}$, to organic hydrocarbon liquids for which the band gap is close to $10 \mathrm{eV} .^{26,27}$ If a reasonably accurate estimate for the band gap is available, Eq. (3) is expected to give the pair formation energy within $20 \%$ accuracy. The pair formation energy of the side chains $\left(E_{p, s c}\right)$ can be approximated by the energy needed to create an electron-hole pair in saturated hydrocarbons $^{27}(25 \mathrm{eV})$. Since the energy deposited in a material by a (pulsed) electron beam is proportional to the electron density in that material, the pair formation energy of a polymer can be calculated according to ${ }^{28}$

$$
E_{p}=\left(\frac{f_{b b}}{E_{p, b b}}+\frac{f_{s c}}{E_{p, s c}}\right)^{-1},
$$

where $f_{b b}$ and $f_{s c}$ denote the fractions of electrons in the backbone and the side chains, respectively.

\section{Time-resolved microwave conductivity}

The change in complex conductivity in the polymer sample after the generation of charges was monitored on a time scale ranging from nanoseconds up to several hundred microseconds by time-resolved microwave conductivity measurements. ${ }^{21}$ The change in complex conductivity due to the generation of charge carriers is given by

$$
\Delta \sigma(\omega)=e n\left[\mu_{h^{+}}(\omega)+\mu_{e^{-}}(\omega)\right],
$$

where $n$ is the number density of electron hole pairs and $\mu_{h^{+}}(\omega)$ and $\mu_{e^{-}}(\omega)$ denote the complex mobility of holes and electrons, respectively. Analogously the complex conductivity can be written as the sum of a real and an imaginary part: $\Delta \sigma(\omega)=\Delta \sigma_{r e}(\omega)+i \Delta \sigma_{i m}(\omega)$. The real part of the imaginary conductivity is due to motion of charges in phase with the probing electric field and can be detected as an increase in the absorption of microwave power in the polymer sample. The motion of charges out of phase with the probing electric field is determined by the imaginary part of the mobility. The imaginary part of the conductivity is related to the permittivity or dielectric constant of the medium $\left(\varepsilon_{o} \varepsilon\right)$ and the radial frequency of the probing electric field according to $\sigma_{i m}(\omega)$ $=\omega \varepsilon_{o} \varepsilon(\omega)$. A change in the imaginary conductivity upon irradiation with a $3 \mathrm{MeV}$ electron pulse gives rise to a decrease in the propagation velocity of the electromagnetic waves through the sample. 

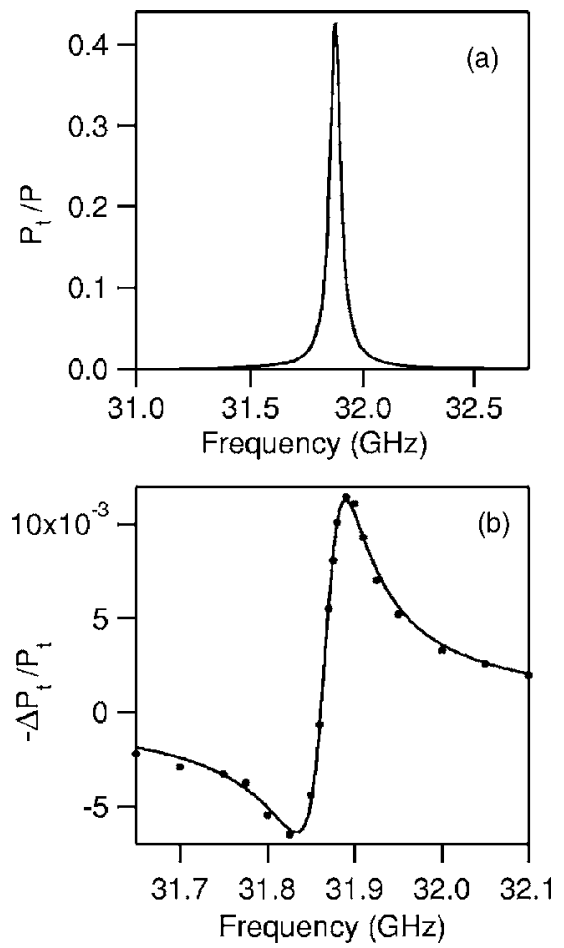

FIG. 3. (a) Measured transmitted microwave power relative to the power directed onto the cell $(P)$ around a resonant frequency for Phe-LPPP with an average chain length of 35 repeat units. (b) Relative change in transmitted microwave power for Phe-LPPP with an average chain length of 35 repeat units. The dots are measured data points recorded $100 \mathrm{~ns}$ after irradiation with a $10 \mathrm{~ns}$ pulse of $3 \mathrm{MeV}$ electrons, the line is a calculated curve with the real and imaginary conductivity as fit parameters.

\section{Microwave resonant cavity}

The use of a microwave resonant cavity enables detection of both the change in real and the change in imaginary conductivity of the sample upon irradiation. ${ }^{29}$ In a resonant transmission cavity setup [see Fig. 2(a)] the electromagnetic waves generated by the microwave source are directed onto the resonant cavity by microwave guides. The polymer sample is placed in a polyethylene container that completely fills the resonant cavity [see Fig. 2(b)]. When the wavelength matches the dimensions of the cavity, resonance occurs and the electromagnetic wave propagates back and forth through the medium in the cavity. The power transmitted by the cavity is detected as a function of frequency [see Fig. 3(a)]. The transmitted power is nonzero around the resonant frequencies of the cavity. The resonant frequencies $\left(f_{\text {res }}\right)$ of the cavity are determined by the cross section $(x \times y)$ and length $(z)$ of the cavity and the propagation velocity of the electromagnetic waves through the sample $(v=c / \sqrt{\varepsilon \kappa}$, where $c$ is the velocity of light in vacuum and $\kappa$ is the relative magnetic permeability of the sample) according to $f_{\text {res }}(k, l, m)$ $=v / 2 \sqrt{(k / x)^{2}+(l / y)^{2}+(m / z)^{2}}$, where $k, l$, and $m$ denote the mode of the electromagnetic wave with resonant frequency $f_{\text {res }}(k, l, m) .{ }^{30}$ The dimensions of the rectangular wave guide $(x=2 y)$ are relatively small as compared to the wavelength of the microwaves generated by the source $(f=26-38 \mathrm{GHz})$.
As a consequence, only the lowest mode of the microwaves can propagate through the wave guide $(k=1, l=0)$, i.e., half the wavelength of the electromagnetic wave in the $x$ direction equals the size of the wave guide in this direction. The resonance curve in Fig. 3(a) is the result for the ladder-type polymer with an average chain length of 35 repeat units $(\varepsilon=2.7, \quad \kappa=1)$ in our resonant cavity $(x \times y \times z=5 \mathrm{~mm}$ $\times 2.5 \mathrm{~mm} \times 24 \mathrm{~mm})$, and corresponds to $m=7$, i.e., the standing wave in the resonant cavity contains seven half wavelengths in the $z$ direction.

The decrease in transmitted microwave power after irradiation of the sample with a $3 \mathrm{MeV}$ electron pulse is a measure for the change in real conductivity. The change in resonant frequency of the cavity upon irradiation is determined by the change in propagation velocity of the electromagnetic waves induced by the change in dielectric constant, and is a measure for the change in imaginary conductivity. Thus, when the charge carriers (generated in a material upon irradiation) exhibit a complex mobility, both a decrease in transmitted power and a shift to lower frequency of the resonance peak is observed. The relative change in the power transmitted by the resonant cavity upon irradiation of the ladder-type polymer with an average chain length of 35 repeat units is shown in Fig. 3(b). From the shape of this curve, qualitative information about the complex conductivity can be deduced. At frequencies slightly lower than the resonant frequency the transmitted power is seen to increase upon the generation of charges, at frequencies slightly higher than the resonance the transmitted power decreases upon irradiation. This indicates a shift of the resonance (to lower frequency). The asymmetry of the change in transmitted microwave power is indicative of an absorption of microwaves upon irradiation in addition to the shift in resonant frequency. Thus, the complex conductivity for the ladder-type polymer with 35 repeat units consists of both an imaginary and a real part. The absolute values of the real and imaginary parts of the complex conductivity can be determined by a fitting procedure which involves a quantitative description of the microwave propagation through the polymer sample. ${ }^{29}$ The result of this analysis is shown by the line in Fig. 3(b).

\section{E. Determination of the complex mobility}

The number density of charge carriers estimated using Eqs. (2)-(4) is the initial charge carrier density; i.e., the total density of charge carriers in the sample created during the $3 \mathrm{MeV}$ electron pulse. The concentration of charges that is of interest for the calculation of the complex mobility [Eq. (5)] is the density of charge carriers that contributes to the complex conductivity signal. The concentration of contributing charges is lower than the initial charge carrier concentration due to the recombination of charges during the electron pulse (i.e., the survival fraction is smaller than 100\%). Therefore we will use the initial charge carrier density as an estimate for the upper limit to the contributing charge carrier density.

The (complex) conductivity measured with TRMC contains contributions of both positive and negative charges (holes and electrons) created during the irradiation of the sample. It is not possible to discriminate between the contri- 

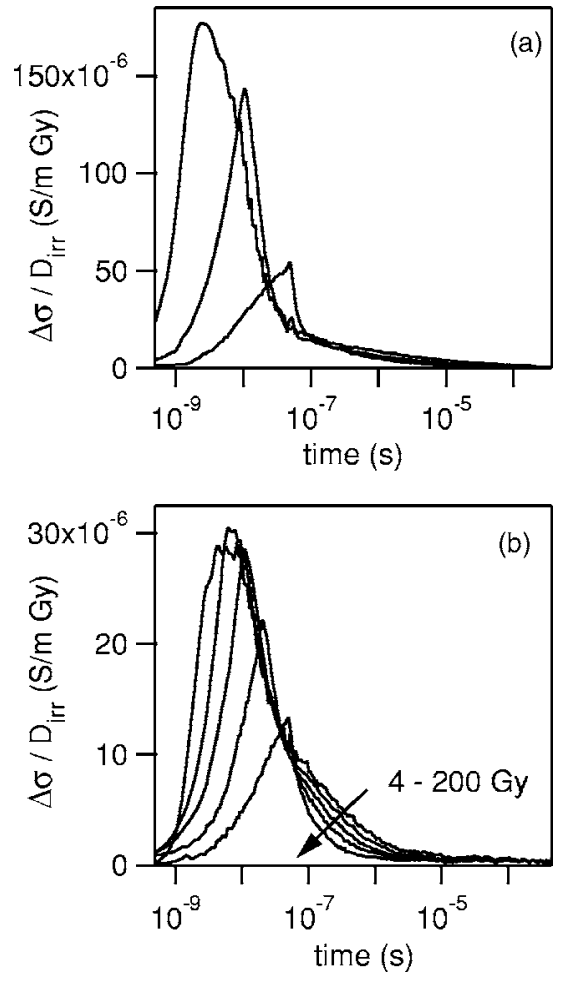

FIG. 4. Dose-normalized change in real conductivity after a $3 \mathrm{MeV}$ electron pulse for an irradiation dose $\left(D_{\text {irr }}\right)$ of 4-200 Gy. (a) Phe ladder polymer with an average chain length of 35 repeat units. (b) Me-ladder polymer with an average chain length of 54 repeat units.

bution of holes and electrons to the complex conductivity from the outcome of our experiments. Therefore, the complex mobility that is calculated from the complex conductivity using Eq. (5) is the sum of the mobility of the hole and the electron.

Thus using the experimentally obtained complex conductivity and the initial charge carrier concentration calculated using Eqs. (2)-(4) in Eq. (5), we derive a lower limit to the sum of the complex mobility for holes and electrons. The mobility obtained is the isotropic mobility $\left(\mu^{\text {iso }}\right)$. The value of interest is the one-dimensional (1D) intrachain mobility $\left(\mu^{1 D}\right)$ i.e., the mobility of the charge moving along the direction of a polymer chain. Taking into account the random orientation of the polymer chains and the linear polarization of the microwave field, these two quantities are related by $\mu^{1 \mathrm{D}}=3 \mu^{\text {iso }} .12$

The real and imaginary parts of the lower limit to the sum of the intrachain hole and electron mobility are denoted by $\Sigma \mu_{\min , r e}^{1 \mathrm{D}}$ and $\Sigma \mu_{\min , i m}^{1 \mathrm{D}}$, respectively.

\section{RESULTS AND DISCUSSION}

\section{A. Dose dependence of the real part of the complex conductivity}

In Fig. 4 the change in complex conductivity for the ladder-type polymer is shown as a function of time after irradiation with a $3 \mathrm{MeV}$ electron pulse with a duration rang- ing from 1-50 ns. The curves in Fig. 4 were recorded at the resonant frequency of the cavity (see Fig. 3). For small changes in the complex conductivity the signal at resonance is not sensitive to the imaginary conductivity, ${ }^{31}$ consequently these curves show the change in real conductivity. The real conductivity increases as charge carriers are created in the polymer sample during irradiation with a $3 \mathrm{MeV}$ electron pulse and decreases after the pulse due to charge carrier recombination or trapping. The signal decays to zero on a time scale of a few microseconds. The maximum conductivity at the end of the pulse is dose dependent, which is indicative of a second order recombination of charges during the pulse and leads to a lower dose-normalized conductivity for higher charge carrier concentrations.

A clear difference is observed in the decay of the conductivity signal after the pulse for the Phe- and Me-ladder-type polymers displayed in Fig. 1. For the Phe-LPPP with an average length of 35 repeat units the decay of the conductivity at longer times $(t>50 \mathrm{~ns})$ is almost independent of the irradiation dose [see Fig. 4(a)], or equivalently, of the concentration of charge carriers. The change in conductivity for Me-LPPP with an average chain length of 54 repeat units is shown in Fig. 4(b). In this case, at high charge carrier density (doses larger than $20 \mathrm{~Gy}$, i.e., initial charge carrier concentration larger than $15 \mu \mathrm{M})$ the conductivity is dosedependent. The larger contribution of second order processes in Me-LPPP leads to a faster decay of the conductivity for Me-LPPP as compared to Phe-LPPP, as can be seen from comparison of Figs. 4(a) and 4(b).

Since both ladder-type polymers in Fig. 1 have the same ribbonlike planar backbone, the difference in decay of the conductivity described above must be attributed to a sidechain effect. The side chains attached to the bridging carbon atom are located relatively far away from the polymer backbone and the $\mathrm{R}_{1}$ substituents (see Fig. 1) consist of linear alkyl chains. Therefore, side-chain effects such as geometrical differences of the polymer backbone due to steric hindrance or differences in electron affinity of the side chains that affect the charge distribution on the backbone, are very unlikely. When interchain interactions affect charge transport, their effect is expected to be more pronounced in MeLPPP than in Phe-LPPP, since the latter polymer has more bulky side chains that are likely to isolate the polymer backbones from neighboring polymer chains. The idea that the conjugated backbones of adjacent Me-LPPP chains are in closer contact than the backbones of Phe-LPPP is consistent with the faster decay observed for Me-LPPP.

\section{B. Time dependence of the complex conductivity}

The change in real and imaginary conductivity after irradiation with a $3 \mathrm{MeV}$ electron pulse is shown in Fig. 5 for the Phe-LPPP with an average chain length of 35 repeat units. The change in real and imaginary conductivity as a function of time after the creation of charges displayed here is obtained from the (time dependent) frequency dependence of the power transmitted by the resonant cavity (see Fig. 3). As described above (see the experiment section) the frequency dependence of the change in microwave power trans- 


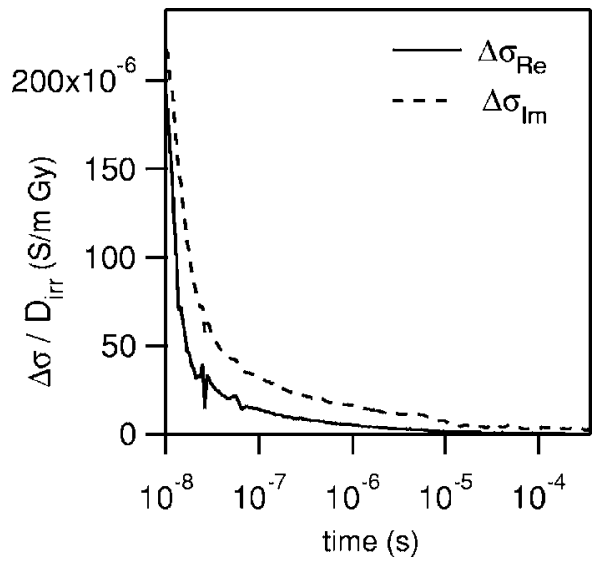

FIG. 5. Real and imaginary dose-normalized conductivity after the creation of charges with a $3 \mathrm{MeV}$ electron pulse for a Pheladder polymer with an average chain length of 35 repeat units at an irradiation dose $\left(D_{i r r}\right)$ of $40 \mathrm{~Gy}$.

mitted by the resonant cavity upon irradiation contains information about the complex conductivity of the sample. As can be seen in Fig. 5, the imaginary part of the complex conductivity is larger than the real part of the complex conductivity for the ladder-type polymer. Any nonzero imaginary part of the complex conductivity is indicative of hindrance of charge transport, as mentioned above. Therefore, the results in Fig. 5 where the imaginary part exceeds the real part of the conductivity show that barriers to charge transport determine the charge transport properties in the ladder-type polymer to a large extent.

\section{Lower limit to the complex mobility}

In order to obtain the complex mobility from the complex conductivity for the ladder-type polymer, the initial charge carrier density was estimated using Eqs. (2)-(4). The band gap of the polymer $(2.7 \mathrm{eV})$ was derived from the onset of optical absorption. ${ }^{32}$ The estimates for the lower limits to the sum of the mobility for holes and electrons obtained with Eq. (5) for the ladder-type polymers are listed in Table I. Both the real and the imaginary parts of the complex mobil-

TABLE I. Lower limit to the real and imaginary parts of the sum of the complex intrachain electron and hole mobility $\left(\Sigma \mu_{\min , r e}^{1 \mathrm{D}}\right.$ and $\left.\Sigma \mu_{\text {min,im }}^{1 \mathrm{D}}\right)$ for ladder-type polymers with an average chain length of $\langle n\rangle$ repeat units, as calculated from the change in complex conductivity using Eqs. (2)-(5). Note that the survival fraction of charges that contributes to the conductivity signal can be different for MeLPPP and Phe-LPPP; therefore, the absolute values of the lower limits to the mobility cannot be compared directly.

\begin{tabular}{lcccc}
\hline \hline LPPP & $\langle n\rangle$ & $\begin{array}{c}\Sigma \mu_{\text {min, re }}^{1 \mathrm{D}} \\
\left(\mathrm{cm}^{2} / \mathrm{V} \mathrm{s}\right)\end{array}$ & $\begin{array}{c}\Sigma \mu_{\text {min, im }}^{1 \mathrm{D}} \\
\left(\mathrm{cm}^{2} / \mathrm{V} \mathrm{s}\right)\end{array}$ & $\frac{\Sigma \mu_{\text {min }, i m}^{1 \mathrm{D}}}{\Sigma \mu_{\text {min, } r e}^{1 \mathrm{D}}}$ \\
\hline Phe & 13 & 0.014 & 0.056 & 4.0 \\
& 16 & 0.021 & 0.080 & 3.8 \\
$\mathrm{Me}$ & 35 & 0.058 & 0.14 & 2.4 \\
\hline \hline
\end{tabular}

ity are found to increase significantly for Phe-LPPP as the average chain length increases from 13-35 repeat units. These results clearly show that limitations in the chain length determine the charge transport properties to a large extent. The chain-length dependence for the lower limit to the real mobility for Phe-LPPP with average chain lengths of 13, 16, and 35 repeat units found here is the same as the dependence derived earlier for the same ladder-type polymers in dilute solutions. ${ }^{20}$ In a dilute solution the polymer chains are isolated from each other by the solvent. Therefore, the intrachain mobility of charges along isolated polymer chains is monitored in TRMC measurements on dilute solutions. ${ }^{12,17,33,34}$ Since the chain-length dependence for the real mobility observed for the ladder-type polymer in solid samples is the same as the chain-length dependence observed for isolated ladder-type polymer chains, we conclude that the intrachain motion of charges is monitored in the experiments on solid samples described here.

The lower limit to the complex mobility for the Me-LPPP with an average length of 54 repeat units is lower than expected from the trend of increasing real and imaginary mobility with the chain length found for Phe-LPPP. This is most likely due to the (over)estimation of the density of charge carriers by taking the initial charge carrier density derived from Eqs. (2)-(4). The observed decay of the conductivity for Me-LPPP is faster than the decay for Phe-LPPP, as discussed above (see Fig. 4). The second order effects during the electron pulse are expected to be more pronounced for Me-LPPP. Therefore, the fraction of the charges initially created during the pulse that contributes to the conductivity signal is smaller for Me-LPPP than for Phe-LPPP. Due to this difference, the lower limits to the complex mobility in the two polymers cannot be compared directly.

The lower limit to the real part of the sum of the electron and hole mobility for the ladder-type polymer with an average chain length of 13 repeat units is already an order of magnitude higher than the device (hole) mobility of $10^{-3} \mathrm{~cm}^{2} / \mathrm{V} \mathrm{s}$ determined by time of flight (TOF) measurements. ${ }^{14}$ This difference can be explained qualitatively by the different measurement techniques used. The high-frequency intrachain mobility deduced from the TRMC measurements cannot be compared directly to the device mobility as determined by space charge limited current (SCLC), in a fieldeffect transistor (FET) or with TOF measurements. In a device set-up, the charges not only have to overcome the injection barriers at the contacts, but the charges also have to migrate over the entire thickness of the sample (which is often in the order of $100 \mathrm{~nm}$ ) to contribute to the mobility signal. Therefore, the mobility of charges determined using a device set-up is sensitive to interchain disorder and limited by charge transport from chain to chain, transport over grain boundaries and over electrode-polymer interfaces. In contrast, in our microwave conductivity measurements we probe the motion of mobile charges along polymer chains and the (complex) conductivity observed is dominated by the most mobile electrons or holes present in the sample.

\section{Absolute value for the ratio of the imaginary and real parts of the complex mobility}

Although absolute values for the complex mobility cannot be derived by the measurement of the complex conductivity 
on solid samples using the TRMC technique, the absolute value for the ratio of the imaginary and the real parts of the complex mobility $\Sigma \mu_{\text {min,im }}^{1 \mathrm{D}} / \Sigma \mu_{\text {min, } r e}^{1 \mathrm{D}}$ can be determined. The ratio decreases with increasing chain length, as can be seen in Table I. This chain-length dependence is observed for both ladder-type polymers irrespective of the side chains, as expected since the ratio is independent of the survival fraction of charge carriers. The chain-length dependence of the ratio directly shows that the out of phase motion of the charge carriers becomes more pronounced as the chain length decreases, i.e., the charge carrier motion is more hindered on shorter chains. This is consistent with the idea that charges moving along a polymer backbone are hindered in their diffusive motion by the ends of the polymer chain.

A clear decrease in the ratio of the imaginary and real mobility is still observed as the average polymer chain length increases from 16 to 35 repeat units. This shows that the charge must diffuse over the entire length of the polymer chain of (at least) 16 repeat units, and encounter a chain end on a time scale in the order of one oscillation period of the microwave field. For one-dimensional diffusion (in the absence of barriers to charge transport), the mean-squared displacement as a function of time is given by $\left\langle x^{2}(t)\right\rangle=2 D t$, where the diffusion constant $D$ is related to the intrachain mobility of the particle performing diffusive motion $\left(\mu_{\text {intra }}\right)$ and temperature according to the Einstein relation $D$ $=\mu_{\text {intra }} k_{B} T / e$. Using a displacement equal to a chain length of 16 repeat units $(14 \mathrm{~nm})$, and a migration time equal to one period of the microwave field $(30 \mathrm{ps})$, a value of $1.3 \mathrm{~cm}^{2} / \mathrm{V} \mathrm{s}$ can be deduced as a lower limit to the intrachain mobility on ladder-type polymers.

\section{DETERMINATION OF THE MOBILITY ALONG INFINITELY LONG LADDER-TYPE POLYMER CHAINS}

In order to obtain the absolute value for the intrachain mobility we describe the motion of charges along the polymer chain by one-dimensional diffusive motion between infinitely high reflecting barriers. In this model, the complex high-frequency intrachain mobility of the charge carriers depends on the radial frequency, the length of the polymer chain $(L)$, and the intrachain mobility (or equivalently the diffusion constant), according to (see the Appendix)

$$
\mu_{a c}(\omega)=8 D \frac{e}{k_{B} T} \sum_{k=0}^{\infty} \frac{\left[c_{k}\right]^{-2}}{\frac{D}{i L^{2} \omega}\left[c_{k}\right]^{2}+1},
$$

where

$$
c_{k}=2 \pi\left(k+\frac{1}{2}\right) .
$$

The chain-length distribution of each of the four ladder-type polymers is known to be a Flory distribution, where the probability of a chain consisting of $n$ monomer units is given by $P(n)=(1-p) p^{n-1}$, where $p=(\langle n\rangle-1) /\langle n\rangle$ denotes the probability of bond formation between adjacent monomer units during the synthesis of the polymer. Chains shorter than five repeat units have been removed by solvent extraction and the presence of chains longer than 75 repeat units is unlikely due to the method of synthesis. ${ }^{23-25}$ To compare the calculated results with the measured data the calculated complex, high-frequency mobility must be averaged over a truncated Flory distribution with $5 \leqslant n \leqslant 75$. The calculated real and imaginary parts of the complex mobility at $30 \mathrm{GHz}$ for the different average chain lengths are shown in Figs. 6(a) and 6(b).

For low intrachain mobility $\left(<0.1 \mathrm{~cm}^{2} / \mathrm{V} \mathrm{s}\right)$, the complex high-frequency mobility is a real quantity and equal to the intrachain mobility; the charge carrier velocity is completely in phase with the oscillating electric field and the motion of the charges is not significantly hindered by the chain ends during the microwave oscillation period. As the intrachain mobility exceeds a few tenths of $\mathrm{cm}^{2} / \mathrm{V} \mathrm{s}$, the imaginary part of the high-frequency mobility is no longer zero and the real part of the high-frequency mobility starts to deviate from the intrachain mobility. These effects arise since the charge carrier starts to encounter the ends of the polymer chain during the microwave period. As the intrachain mobility increases further to tens of $\mathrm{cm}^{2} / \mathrm{V} \mathrm{s}$, the chain ends strongly affect the high-frequency mobility. This results in a decrease of the real part of the high-frequency mobility with increasing intrachain mobility and a further increase of the imaginary part of the high-frequency mobility. At high intrachain mobility $\left(>1000 \mathrm{~cm}^{2} / \mathrm{V} \mathrm{s}\right)$ the complex high-frequency mobility is completely imaginary: The charge carrier velocity is completely out of phase with the oscillating electric field, as a result to frequent hindrance of the charge carrier motion at the polymer chain ends.

The value of the intrachain mobility can be obtained by comparison of the calculated ratio of the imaginary and the real parts of the complex high frequency mobility $\mu_{a c, i m} / \mu_{a c, r e}$ with the experimental results. The calculated curves for the ratio $\mu_{a c, i m} / \mu_{a c, \text { re }}$ for the four average chain lengths in Fig. 6(c) show that the experimentally determined ratios (denoted by the dots) are reproduced by an intrachain mobility close to $30 \mathrm{~cm}^{2} / \mathrm{V} \mathrm{s}$. This agrees with the estimate made above, that the intrachain mobility must exceed $1.5 \mathrm{~cm}^{2} / \mathrm{V} \mathrm{s}$ in order to explain the chain-length dependence of the experimental results.

Comparison of the experimentally determined lower limit to the real and imaginary parts of the sum of the intrachain mobility for electrons and holes at high frequency as denoted in Table I, with the calculated values for the real and imaginary parts of the complex high-frequency mobility [Eq. (6)] at a intrachain mobility of $30 \mathrm{~cm}^{2} / \mathrm{V} \mathrm{s}$ gives the percentage of the initially created charges that contribute to the TRMC conductivity signal. Survival fractions of $3.7 \%$ and $1.3 \%$ are found for Phe-LPPP and Me-LPPP, respectively. The real and imaginary parts of the high-frequency charge carrier mobility calculated for these survival fractions are denoted by dots in Figs. 6(a) and 6(b). The larger charge carrier yield found for Phe-LPPP with respect to Me-LPPP is consistent with the different decay kinetics for these two polymers, as discussed above.

The value of $30 \mathrm{~cm}^{2} / \mathrm{V} \mathrm{s}$ found for the intrachain mobility is several orders of magnitude higher than the device (hole) mobility of $10^{-3} \mathrm{~cm}^{2} / \mathrm{V} \mathrm{s}$ determined by TOF 

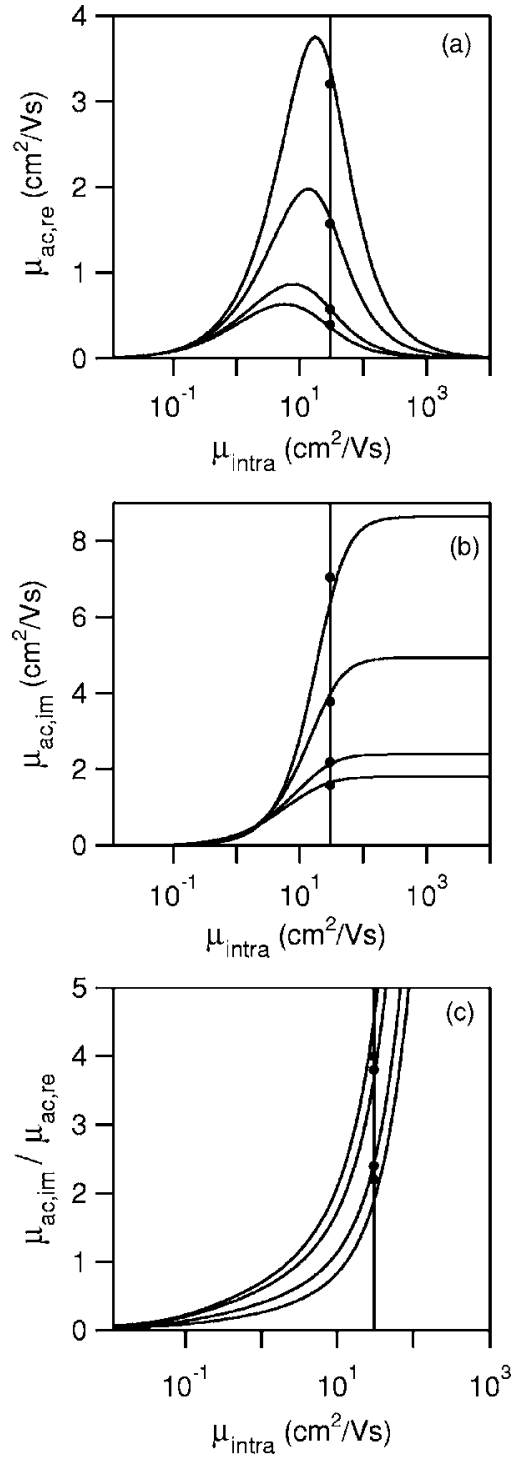

FIG. 6. (a) Real $\left(\mu_{a c, r e}\right)$ and (b) imaginary $\left(\mu_{a c, i m}\right)$ parts of the complex high-frequency mobility as a function of intrachain mobility calculated with Eq. (6) at $30 \mathrm{GHz}$, for ladder-type polymers with an average number of repeat units of $13,16,35$, and 54 , from bottom to top. (c) Ratio of the imaginary and the real highfrequency mobility $\mu_{a c, i m} / \mu_{a c, r e}$ calculated with Eq. (6) for $30 \mathrm{GHz}$, for ladder-type polymers with an average number of repeat units of $13,16,35$, and 54, from top to bottom. The experimentally determined ratios shown in Table I, represented by dots, must be reproduced by a single intrachain mobility for the four chain lengths. The experimental values for the ratio $\Sigma \mu_{\text {min,im }}^{1 \mathrm{D}} / \Sigma \mu_{\text {min,re }}^{1 \mathrm{D}}$ are reproduced by taking a intrachain mobility near $30 \mathrm{~cm}^{2} / \mathrm{V} \mathrm{s}$ (indicated with the vertical line). The dots in (a) and (b) indicate the real and imaginary parts of the complex mobility for the four chain lengths calculated from the complex conductivity with a survival fraction of $3.7 \%$ for Phe-LPPP and $1.3 \%$ for Me-LPPP.

measurements. ${ }^{14}$ This difference can be explained by the device-specific properties and intrinsic material properties that limit the device mobility, as mentioned above.

We can now compare the intrachain mobility for charge carriers on ladder-type polymer chains in solid samples found in the present work with the intrachain mobility deter- mined previously for isolated ladder-type polymer chains in dilute solutions. ${ }^{20}$ Because of the rigid structure of the ladder-type polymer the conformation of the polymer backbone is expected to be identical in these two experiments. The comparison of charge transport on isolated ladder-type polymer chains to the intrachain charge transport for the same polymer in an environment where interchain effects can play a role, gives information about the effect of intermolecular interactions on the intrachain charge transport properties. The value for the intrachain mobility for (positive) charges on isolated chains of the ladder-type polymer in a dilute solution was found to be $600 \mathrm{~cm}^{2} / \mathrm{V} \mathrm{s},{ }^{20}$ i.e., a factor of 20 higher than the value found for solid samples. This difference in intrachain mobility shows that the disorder induced by the presence of neighboring polymer chains reduces the charge carrier motion significantly. Interchain interactions cause energy variations along a polymer backbone. Thus a mobile charge carrier encounters a disordered energy landscape which leads to a lower intrachain mobility.

\section{SUMMARY AND CONCLUSIONS}

We have shown that the ratio between the real and imaginary parts of the complex high-frequency mobility found for ladder-type polymers strongly depends on the length of the polymer chain. Therefore, it can be concluded that the measurement of the complex charge carrier mobility gives a unique insight into the effect of the conjugation length on the intrachain mobility of charge carriers along semiconducting polymers.

The experimental results can be described by onedimensional diffusive motion along the polymer backbone. The intrachain mobility found for ladder-type polymer chains subject to interchain interactions is $30 \mathrm{~cm}^{2} / \mathrm{V} \mathrm{s}$, which is 20 times lower than the mobility for charges along isolated ladder-type polymer chains found earlier. Thus interchain interactions are found to severely decrease the intrachain charge carrier mobility. However, the intrachain mobility found in the present work is more than four orders of magnitude higher than mobility values obtained earlier from time of flight measurements. Hence, ladder-type polymers are very promising materials for optoelectronic applications provided that interchain effects on the charge carrier mobility can be minimized.

\section{ACKNOWLEDGMENTS}

This work was part of the research program of the Stichting voor Fundamenteel Onderzoek der Materie (FOM), which is financially supported by the Nederlandse Organisatie voor Wetenschappelijk Onderzoek (NWO).

\section{APPENDIX: ONE-DIMENSIONAL DIFFUSIVE MOTION BETWEEN INFINITELY HIGH REFLECTING BARRIERS}

The one-dimensional diffusive motion of a charge carrier on a polymer chain can be described by the partial differen- 
tial equation that gives the dependence of charge density $(C)$ as a function of time $(t)$ and place $(x)$

$$
\frac{\partial C(x, t)}{\partial t}=D \frac{\partial^{2} C(x, t)}{\partial x^{2}}
$$

where $D$ is the one-dimensional diffusion constant of a charge on the polymer chain. To derive the charge density as a function of time and place we solve Eq. (A1) while taking into account the following boundary conditions.

(1) The chain ends located at $x=0$ and $x=L$ act as infinitely high reflecting barriers to charge transport

$$
\begin{aligned}
& \left.\frac{\partial C(x, t)}{\partial x}\right|_{x=0}=0, \\
& \left.\frac{\partial C(x, t)}{\partial x}\right|_{x=L}=0 .
\end{aligned}
$$

(2) The charge is initially localized at a position $q$ along the polymer chain.

$$
C(x, 0)=\delta(x-q) \text {. }
$$

The charge density as a function of time and place is then given by

$$
C(x, t)=\frac{1}{L} \sum_{k=-\infty}^{\infty} \cos \frac{\pi k x}{L} \cos \frac{\pi k q}{L} e^{-(\pi k / L)^{2} D t} .
$$

This can easily be verified by substituting this solution in the differential equation in Eq. (A1), and by confirming that the boundary conditions [Eqs. (A2) and (A3)] are fulfilled.

The mean-squared displacement of the charge as a function of time and the initial site of the charge carrier is now given by

$$
\begin{aligned}
\left\langle(x-q)^{2}\right\rangle \equiv & \int_{0}^{L} d x(x-q)^{2} C(x, t) \\
= & \frac{L^{2}}{3}-L q+q^{2}+4 \sum_{k=1}^{\infty}\left(\frac{L}{\pi k}\right)^{2} \\
& \times\left(\cos \frac{\pi k q}{L}\right)\left\{\left(1-\frac{q}{L}\right)(-1)^{k}+\frac{q}{L}\right\} e^{-(\pi k / L)^{2} D t} .
\end{aligned}
$$

To obtain the mean-squared displacement of the charge as a function of time we assume that the initial position of the charge carrier is uniformly distributed along the polymer chain, thus we average Eq. (A5) over all initial sites, i.e., $q$ $\in[0, L]$

$$
\begin{aligned}
\overline{\left\langle(x-q)^{2}\right\rangle} & \equiv L^{-1} \int_{0}^{L} d q\left\langle(x-q)^{2}\right\rangle \\
& =L^{2}\left\{\frac{1}{6}-\sum_{k=0}^{\infty}\left(\frac{2}{c_{k}}\right)^{4} e^{-\left(c_{k} / L\right)^{2} D t}\right\},
\end{aligned}
$$

where $c_{k}=2 \pi\left(k+\frac{1}{2}\right)$.

The (complex) frequency dependent diffusion coefficient $D_{a c}(\omega)$ that describes the motion of charge carriers along a polymer chain with infinitely high barriers to charge transport at the chain ends can now be derived according to Kubo's formula: ${ }^{22}$

$$
D_{a c}(\omega) \equiv-\frac{\omega^{2}}{2} \int_{0}^{\infty} \overline{\left\langle(x-q)^{2}\right\rangle} e^{-i \omega t} d t=8 D \sum_{k=0}^{\infty} \frac{c_{k}^{-2}}{1+\frac{c_{k}^{2} D}{i \omega L^{2}}}
$$

The complex frequency dependent intrachain mobility of the charge carriers given in Eq. (6) in the main text follows from this expression by taking into account the Einstein relation that relates the diffusion coefficient to the charge carrier mobility:

$$
D_{a c}=\frac{k_{B} T}{e} \mu_{a c}
$$

${ }^{1}$ L. L. Chua, J. Zaumsell, J. F. Chang, E. C. W. Ou, P. K. H. Ho, H. Sirringhaus, and R. H. Friend, Nature (London) 434, 194 (2005).

${ }^{2}$ H. Sirringhaus, N. Tessler, and R. H. Friend, Science 280, 1741 (1998).

${ }^{3}$ H. E. A. Huitema, G. H. Gelinck, J. B. P. H. van der Putten, K. E. Kuijk, C. M. Hart, E. Cantatore, P. T. Herwig, A. J. J. M. van Breemen, and D. M. de Leeuw, Nature (London) 414, 599 (2001).

${ }^{4}$ H. E. A. Huitema, G. H. Gelinck, J. B. P. H. van der Putten, K. E. Kuijk, K. M. Hart, E. Cantatore, and D. M. de Leeuw, Adv. Mater. (Weinheim, Ger.) 14, 1201 (2002).

${ }^{5}$ F. Hide, M. A. Diaz-Garcia, B. J. Schwartz, and A. J. Heeger,
Acc. Chem. Res. 30, 430 (1996).

${ }^{6}$ I. Heller, J. Kong, H. A. Heering, K. A. Williams, S. G. Lemay, and C. Dekker, Nano Lett. 5, 137 (2004).

${ }^{7}$ R. L. Carroll and C. B. Gorman, Angew. Chem. 41, 4378 (2002).

${ }^{8}$ C. Joachim, J. K. Gimzewske, and A. Aviram, Nature (London) 408, 541 (2000).

${ }^{9}$ S. Kubatkin, A. Danilov, M. Hjort, J. Cornil, J. Bredas, N. SturhHansen, P. Hedegard, and T. Bjornholm, Curr. Appl. Phys. 4, 554 (2004).

${ }^{10}$ M. Lögdlund and W. R. Salaneck, in Semiconducting Polymers, edited by G. Hadziioannou and P. F. van Hutten (Wiley-vch, Weinheim, 2000), p. 115.

${ }^{11}$ D. L. Lide, CRC Handbook of Chemistry and Physics (CRC 
Press, Boca Raton, FL, 1991).

${ }^{12}$ P. Prins, L. P. Candeias, A. J. J. M. van Breemen, J. Sweelssen, P. T. Herwig, H. F. M. Schoo, and L. D. A. Siebbeles, Adv. Mater. (Weinheim, Ger.) 17, 718 (2005).

${ }^{13}$ P. W. M. Blom, M. J. M. de Jong, and J. J. M. Vleggaar, Appl. Phys. Lett. 68, 3308 (1996).

${ }^{14}$ D. Hertel, H. Bässler, U. Scherf, and H. H. Hörhold, J. Chem. Phys. 110, 9214 (1999).

${ }^{15}$ H. Sirringhaus, P. Brown, R. H. Friend, M. M. Nielsen, K. Bechgaard, B. M. W. Langeveld-Voss, A. J. H. Spiering, R. A. J. Janssen, E. W. Meijer, P. Herwig, and D. M. de Leeuw, Nature (London) 401, 685 (1999).

${ }^{16}$ H. Ishii, K. Sugiyama, E. Ito, and K. Seki, Adv. Mater. (Weinheim, Ger.) 11, 605 (1999).

${ }^{17}$ F. C. Grozema, L. D. A. Siebbeles, J. M. Warman, S. Seki, S. Tagawa, and U. Scherf, Adv. Mater. (Weinheim, Ger.) 14, 228 (2002).

${ }^{18}$ F. C. Grozema, P. T. van Duijnen, Y. A. Berlin, M. A. Ratner, and L. D. A. Siebbeles, J. Phys. Chem. B 106, 7791 (2002).

${ }^{19}$ L. P. Candeias, F. C. Grozema, G. Padmanaban, S. Ramakrishnan, L. D. A. Siebbeles, and J. M. Warman, J. Phys. Chem. B 107, 1554 (2003).

${ }^{20}$ P. Prins, F. Grozema, J. Schins, S. Patil, U. Scherff, and L. Siebbeles (unpublished).

${ }^{21}$ P. P. Infelta, M. P. de Haas, and J. Warman, Radiat. Phys. Chem. 10, 353 (1977).

${ }^{22}$ R. Kubo, J. Phys. Soc. Jpn. 12, 570 (1957).
${ }^{23}$ U. Scherf, J. Mater. Chem. 9, 1853 (1999).

${ }^{24}$ U. Scherf, A. Bohnen, and K. Müllen, Macromol. Chem. Phys. 193, 1127 (1992).

${ }^{25}$ U. Scherf and K. Müllen, Makromol. Chem., Macromol. Symp. 12, 489 (1991).

${ }^{26}$ R. C. Alig, S. Bloom, and C. W. Struck, Phys. Rev. B 22, 5565 (1980).

${ }^{27}$ A. Hummel, in The Chemistry of Alkanes and Cycloalkanes, edited by S. Patai and Z. Rappoport (Wiley, New York, 1992), p. 743.

${ }^{28}$ G. H. Gelinck and J. M. Warman, J. Phys. Chem. 100, 20035 (1996).

${ }^{29}$ J. M. Schins, P. Prins, F. C. Grozema, R. D. Abellon, M. P. de Haas, and L. D. A. Siebbeles, Rev. Sci. Instrum. 76, 8 (2005).

${ }^{30}$ E. R. Dobbs, Basic Electromagnetism (Chapman and Hall, London, 1993).

${ }^{31}$ J. J. Piet, in Excitonic Interactions in Multichromophoric Arrays (Radiation Chemistry Department, Delft University of Technology, Delft, 2001).

${ }^{32}$ J. Stampfl, W. Graupner, G. Leising, and U. Scherf, J. Lumin. 63, 117 (1995).

${ }^{33}$ F. C. Grozema, R. J. O. M. Hoofman, L. P. Candeias, M. P. de Haas, J. M. Warman, and L. D. A. Siebbeles, J. Phys. Chem. A 107, 5976 (2003).

${ }^{34}$ R. J. O. M. Hoofman, M. P. de Haas, L. D. A. Siebbeles, and J. M. Warman, Nature (London) 392, 54 (1998). 